PROCEEDINGS OF THE

AMERICAN MATHEMATICAL SOCIETY

Volume 125, Number 4, April 1997, Pages 1187-1192

S 0002-9939(97)03694-0

\title{
RUDIN'S ORTHOGONALITY PROBLEM AND THE NEVANLINNA COUNTING FUNCTION
}

\author{
PAUL S. BOURDON
}

(Communicated by Theodore W. Gamelin)

\begin{abstract}
Let $\phi$ be a holomorphic function taking the open unit disk $U$ into itself. We show that the set of nonnegative powers of $\phi$ is orthogonal in $L^{2}(\partial U)$ if and only if the Nevanlinna counting function of $\phi, N_{\phi}$, is essentially radial. As a corollary, we obtain that the orthogonality of $\left\{\phi^{n}: n=0,1,2, \ldots\right\}$ for a univalent $\phi$ implies $\phi(z)=\alpha z$ for some constant $\alpha$. We also show that if $\left\{\phi^{n}: n=0,1,2, \ldots\right\}$ is orthogonal, then the closure of $\phi(U)$ must be a disk.
\end{abstract}

\section{INTRODUCTION}

Observe that if $f$ is an inner function on the open unit disk $U$ satisfying $f(0)=0$, then $\left\{f^{n}: n=0,1,2, \ldots\right\}$ is orthogonal in $L^{2}(\partial U)$. The following problem was posed by Walter Rudin in 1988 (at an MSRI conference):

Question 1 (Rudin). If $f$ is a bounded analytic function on the open unit disk $U$ such that $\left\{f^{n}: n=0,1,2, \ldots\right\}$ is orthogonal in $L^{2}(\partial U)$, must $f$ be a constant multiple of an inner function?

Note that if the $H^{\infty}$ function $f$ satisfies the orthogonality condition of Rudin's question, then $f(0)=0$. Also note that any constant multiple of $f$ will satisfy the condition; thus, we may restrict our attention to maps $f$ taking $U$ into itself. For the remainder of this paper, $\phi$ will denote an analytic map taking $U$ into $U$.

Suppose that $\phi$ is inner and fixes the origin; then according to Littlewood's Inequality (discussed in the next section), the Nevanlinna counting function of $\phi$, $N_{\phi}$, is given by $N_{\phi}(w)=\log (1 /|w|)$ for all $w$ outside of some subset of $U$ having logarithmic capacity 0 . Thus, $N_{\phi}$ is essentially a radial function when $\phi$ is an inner function vanishing at zero. In this paper, we prove that Rudin's orthogonality problem is equivalent to the following.

Question 2. Suppose that for almost every $r \in[0,1], \theta \mapsto N_{\phi}\left(r e^{i \theta}\right)$ is essentially constant; must $\phi$ be a constant multiple of an inner function?

That the preceding question is equivalent to Rudin's Question 1 follows from our Main Theorem.

Received by the editors October 27, 1995.

1991 Mathematics Subject Classification. Primary 30D50.

The author's research was supported in part by the National Science Foundation (DMS 9401206). 
Main Theorem. Let $\phi$ be a self-map of $U$ fixing 0 . The set $\left\{\phi^{n}: n=0,1,2, \ldots\right\}$ is orthogonal in $L^{2}(\partial U)$ if and only if there is a function $g:[0,1] \rightarrow[0, \infty)$ such that for almost every $r \in[0,1], N_{\phi}\left(r e^{i \theta}\right)=g(r)$ for almost every $\theta \in[0,2 \pi]$.

We obtain as corollaries of the Main Theorem two results that suggest that the answer to Rudin's question is "yes". First we show the answer is "yes" if $\phi$ is assumed to be univalent (see Corollary 1). Then we show that if $\phi$ satisfies the orthogonality hypothesis, the closure of $\phi(U)$ must be a disk (see Corollary 2). Cima, Korenblum, and Stessin [2] have also obtained evidence that Rudin's question has an affirmative answer: they show that if $f$ is in the disk algebra with boundary function in $\operatorname{Lip}_{\alpha}$ for some $\alpha>1 / 2$ and if the set of nonnegative powers of $f$ is orthogonal in $L^{2}(\partial U)$, then $f$ must be a multiple of an inner function. They also obtain a result similar to our Corollary 2 (see [2, Corollary 1$]$ ).

\section{BACKGROUND}

A bounded analytic function on $U$ has finite radial limit at almost every point on the boundary of $U$ (see, e.g., [3, Theorem 1.3]). If the radial limit function has modulus 1 almost everywhere, then the function is said to be inner.

Let $f$ and $g$ be bounded and analytic on $U$. The inner product of $f$ and $g$ in $L^{2}(\partial U)$, which we will denote $\langle f, g\rangle$, may be computed in several ways:

$$
\begin{aligned}
\langle f, g\rangle & =\frac{1}{2 \pi} \int_{0}^{2 \pi} f\left(e^{i \theta}\right) \overline{g\left(e^{i \theta}\right)} d \theta \\
& =\sum_{k=0}^{\infty} \hat{f}(n) \overline{\hat{g}(n)} \\
& =\frac{1}{\pi} \int_{U} f^{\prime}(z) \overline{g^{\prime}(z)} \log \left(1 /|z|^{2}\right) d A(z)+f(0) \overline{g(0)} .
\end{aligned}
$$

In the first representation of the inner product, $f\left(e^{i \theta}\right)$ and $g\left(e^{i \theta}\right)$ denote, respectively, the radial limits of $f$ and $g$ at $e^{i \theta}$. In the second, $\hat{f}(n)$ and $\hat{g}(n)$ denote, respectively, the $n$-th Taylor coefficients of $f$ and $g$ in the expansion about the origin. The third representation is the Littlewood-Paley formula, which may be proved using power series ( $d A$ represents area measure).

The first step in our proof of the Main Theorem entails the use of the third representation of the inner product followed by a change of variables. The change of variables introduces the Nevanlinna counting function. For a holomorphic map $\phi$ taking $U$ into itself, the Nevanlinna counting function of $\phi, N_{\phi}$, is defined on $U \backslash\{\phi(0)\}$ by

$$
N_{\phi}(w)=\sum_{\phi(z)=w} \log (1 /|z|),
$$

where multiplicities are counted and $N_{\phi}(w)$ is taken to be zero if $w$ is not in the range of $\phi$. The Nevanlinna counting function of $\phi$ is lower semicontinuous and satisfies the sub-mean-value property ([5, Section 2$])$. Some other important properties of $N_{\phi}$ are described in the following result due to Littlewood ([6], see also [8, $4.2])$.

Littlewood's Inequality. For each $w \in U \backslash\{\phi(0)\}$,

$$
N_{\phi}(w) \leq \log \left|\frac{1-\overline{\phi(0)} w}{\phi(0)-w}\right| ;
$$


moreover, the following are equivalent:

- equality occurs in $(\dagger)$ at some $w$ in $U$;

- equality occurs in $(\dagger)$ at all points in $U$ outside a subset of $U$ having zero logarithmic capacity;

- $\phi$ is an inner function.

The preceding result shows that inner functions that vanish at 0 have Nevanlinna counting functions that are essentially radial. As we pointed out in the introduction, our work shows that Rudin's orthogonality problem is equivalent to "If $N_{\phi}$ is radial, must $\phi$ be inner?"

\section{Results}

The following lemma is an easy consequence of the Müntz-Szasz Theorem (see, e.g., [7, p. 336]; see also the note added in proof at the end of this paper).

Müntz-Szasz Lemma. Suppose that $0<\lambda_{1}<\lambda_{2}<\lambda_{3}<\cdots$ and $\sum_{k=1}^{\infty} 1 / \lambda_{k}$ diverges. Then the set of all finite linear combinations of the functions defined on $[0,1]$ by

$$
t \mapsto t^{\lambda_{1}}, t \mapsto t^{\lambda_{2}}, t \mapsto t^{\lambda_{3}}, \ldots
$$

is dense in $L^{2}([0,1])$.

Proof. By the Müntz-Szasz Theorem, the set of all finite linear combinations of

$$
t \mapsto 1, t \mapsto t^{\lambda_{1}}, t \mapsto t^{\lambda_{2}}, t \mapsto t^{\lambda_{3}}, \ldots
$$

is dense in $C([0,1])$ (where, as usual, $C([0,1])$ denotes the set of continuous, complex-valued functions on $[0,1]$ with the supremum norm). It follows that any continuous function on $[0,1]$ that vanishes at 0 may be uniformly approximated by functions in the linear span of $\left\{t \mapsto t^{\lambda_{1}}, t \mapsto t^{\lambda_{2}}, t \mapsto t^{\lambda_{3}}, \ldots\right\}$. However, the set of continuous functions vanishing at 0 is dense in $L^{2}([0,1])$.

Main Theorem. Let $\phi$ be a self-map of $U$ fixing 0 . The following are equivalent:

(a) the set $\left\{\phi^{n}: n=0,1,2, \ldots\right\}$ is orthogonal in $L^{2}(\partial U)$;

(b) there is a function $g:[0,1] \rightarrow[0, \infty)$ such that for almost every $r \in[0,1]$, $N_{\phi}\left(r e^{i \theta}\right)=g(r)$ for almost every $\theta \in[0,2 \pi]$.

Proof. Suppose (b) holds. Let $n>m$ be nonnegative integers and let $d \lambda=d A / \pi$. We have

$$
\begin{aligned}
\left\langle\phi^{n}, \phi^{m}\right\rangle & =n m \int_{U} \phi(z)^{n-1} \overline{\phi(z)^{m-1}}\left|\phi^{\prime}(z)\right|^{2} \log \left(1 /|z|^{2}\right) d \lambda(z) \\
& =2 n m \int_{U} z^{n-1} \overline{z^{m-1}} N_{\phi}(z) d \lambda(z) \\
& =2 n m \int_{0}^{1} r^{n+m-1}\left[\int_{0}^{2 \pi} e^{i(n-m) \theta} N_{\phi}\left(r e^{i \theta}\right) d \theta\right] d r \\
& =2 n m \int_{0}^{1} r^{n+m-1} g(r)\left[\int_{0}^{2 \pi} e^{i(n-m) \theta} d \theta\right] d r \\
& =0 .
\end{aligned}
$$

The change of variables yielding the second equality is justified in, e.g., [8, 4.3]. 
Conversely, suppose $\left\{\phi^{n}: n=0,1,2, \ldots\right\}$ is orthogonal. Let $k$ be an arbitrary positive integer. For each integer $n$ larger than $k$, we have

$$
\begin{aligned}
0 & =\left\langle\phi^{n}, \phi^{n-k}\right\rangle \\
& =n(n-k) \int_{U} \phi(z)^{n-1} \overline{\phi(z)^{n-k-1}}\left|\phi^{\prime}(z)\right|^{2} \log \left(1 /|z|^{2}\right) d \lambda(z) \\
& =2 n(n-k) \int_{U} z^{n-1} \overline{z^{n-k-1}} N_{\phi}(z) d \lambda(z) \\
& =2 n(n-k) \int_{0}^{1} r^{2 n-k-1}\left[\int_{0}^{2 \pi} e^{i k \theta} N_{\phi}\left(r e^{i \theta}\right) d \theta\right] d r .
\end{aligned}
$$

Thus the function $f_{k}(r):=\int_{0}^{2 \pi} e^{-i k \theta} N_{\phi}\left(r e^{i \theta}\right) d \theta$ is orthogonal in $L^{2}([0,1])$ to the linear span of the set $\left\{r \mapsto r^{2 n-k-1}: n>k\right\}$ (Littlewood's inequality shows that $\left|f_{k}(r)\right| \leq 2 \pi \log (1 / r)$, so $f_{k}$ is in $\left.L^{2}([0,1])\right)$. By the Muntz-Szasz Lemma, the linear span of $\left\{r \mapsto r^{2 n-k-1}: n>k\right\}$ is dense in $L^{2}([0,1])$; hence, $f_{k}(r)=0$ for almost every $r$ in $[0,1]$. Taking complex conjugates, we also have that $f_{-k}$ vanishes at almost every point of $[0,1]$. Since $k$ is an arbitrary positive integer, we may conclude that there is a set $E$ of full measure in $[0,1]$ such that for each $r \in E$,

$$
\int_{0}^{2 \pi} e^{i j \theta} N_{\phi}\left(r e^{i \theta}\right) d \theta=0 \text { for all } j \neq 0 .
$$

Thus for fixed $r \in E$, we see that $\theta \mapsto N_{\phi}\left(r e^{i \theta}\right)$ is essentially constant, equaling, say, $g(r)$. Set $g(r)=0$ for $r \in[0,1] \backslash E$, and the proof is complete.

The following two corollaries provide some evidence that the answer to Rudin's orthogonality question is "yes".

Corollary 1. Suppose that $\phi$ is a univalent self-map of $U$ the set of whose nonnegative powers is orthogonal in $L^{2}(\partial U)$. Then there is a constant $\alpha$ such that $\phi(z)=\alpha z$.

Proof. We know $\phi(0)=0$ because $\phi^{1}$ is orthogonal to $\phi^{0}$. Let $D$ be a disk centered at 0 and contained in $\phi(U)$. On $D \backslash\{0\}$,

$$
N_{\phi}(w)=\log \left(1 /\left|\phi^{-1}(w)\right|\right)
$$

so that $N_{\phi}$ is continuous (in fact, harmonic) on $D \backslash\{0\}$. By continuity and the Main Theorem, we see that $N_{\phi}$ is a radial function on $D \backslash\{0\}$. Because $N_{\phi}$ is radial and harmonic on $D \backslash\{0\}$, there are constants $b$ and $c$ such that on $D \backslash\{0\}$

$$
N_{\phi}(w)=b \log |1 / w|+c
$$

(see, e.g., [1, Lemma 3.10]). Exponentiating both sides of the equation above and composing with $\phi$, we have for each $z \in \phi^{-1}(D \backslash\{0\})$

$$
\frac{1}{|z|}=\left|\frac{1}{\phi(z)}\right|^{b} e^{c}
$$

Observe that

$$
\lim _{z \rightarrow 0}|\phi(z)|^{b-1}=e^{c} /\left|\phi^{\prime}(0)\right| ;
$$

and because $\phi(0)=0$, it follows that $b=1$. Thus

$$
\left|\frac{\phi(z)}{z}\right|=e^{c}
$$


for $z$ near 0 , and thus $\phi(z) / z$ must be constant near zero, hence constant on all of $U$.

Corollary 2. Suppose that $\phi$ is a self-map of $U$ the set of whose nonnegative powers is orthogonal in $L^{2}(\partial U)$. Then the closure of $\phi(U)$ is a disk.

Proof. If $\phi$ is constant, then $\phi \equiv 0$ and $\phi(U)=\{0\}$. Assume that $\phi$ is nonconstant. We know that there is a set $E \subset[0,1]$ of measure 1 such that for each $r \in E$, $N_{\phi}\left(r e^{i \theta}\right)$ is essentially constant in $\theta$. Let $r \in E$. If the range of $\phi$ contains a point on the circle $C_{r}:=\left\{r e^{i \theta}: \theta \in[0,2 \pi]\right\}$ (hence an entire interval by the open mapping theorem), then the range of $\phi$ may omit only a $\theta$-measure-zero subset of $C_{r}$ because

(1) $N_{\phi}$ is essentially constant on $C_{r}$, and

(2) $N_{\phi}(w)=0$ if and only if $w$ is not in the range of $\phi$.

Thus if the range hits $C_{r}$, the closure of the range must contain $C_{r}$. Hence the closure of the range contains $C_{r}$ for $r \in E \cap\left[0,\|\phi\|_{\infty}\right)$. Because $E$ has full measure in $[0,1]$, the closure of the range must be the closed disk of radius $\|\phi\|_{\infty}$.

Using different methods, Stephen Fisher has obtained a result stronger than the preceding corollary: he has shown that if the set of nonnegative powers of $\phi$ is orthogonal, then $\phi(U)$ must be a disk except possibly for a set of logarithmic capacity zero. We conclude this note with a proof of Fisher's result based on the Main Theorem.

Theorem (Fisher). Suppose that $\phi$ is a self-map of $U$ the set of whose nonnegative powers is orthogonal in $L^{2}(\partial U)$; suppose also that $\|\phi\|_{\infty}=1$. Then there is a subset $E$ of $U$ having logarithmic capacity zero such that $\phi(U)=U \backslash E$.

Proof. There is a subset $E$ of $U$ having capacity zero such that on $U \backslash E$ the function $N_{\phi}$ is continuous (see, e.g., p. 327 of [4]). That $N_{\phi}$ is radial on $U \backslash E$ is an easy consequence of our Main Theorem - thus, if $w_{1}$ and $w_{2}$ are points of continuity of $N_{\phi}$ with $\left|w_{1}\right|=\left|w_{2}\right|$, we have $N_{\phi}\left(w_{1}\right)=N_{\phi}\left(w_{2}\right)$.

Suppose that $\phi(U)$ omits a subset $H$ of $U$ having positive capacity. Let $w_{0}$ be a point of continuity of $N_{\phi}$ contained in $H$. Because $w_{0}$ is not in the range of $\phi$, we have $N_{\phi}\left(w_{0}\right)=0$. Because $w_{0}$ is a point of continuity of $N_{\phi}$, we see that $N_{\phi}(w)=0$ at each point $w$ of continuity with $|w|=\left|w_{0}\right|$. Now observe that there must be a point on the circle of radius $\left|w_{0}\right|$ that is in $\phi(U)(\phi(0)=0$ by the orthogonality condition, and, by hypothesis, $\left.\|\phi\|_{\infty}=1\right)$. Because $\phi$ is an open mapping, $\phi(U)$ contains a nonempty open arc $J$ of points on the circle $\left\{z:|z|=\left|w_{0}\right|\right\}$. This arc $J$ has positive capacity and hence contains a point of continuity of $N_{\phi}$; at this point of continuity we must have $N_{\phi}=0$, but this contradicts the fact that every point of $J$ lies in $\phi(U)$.

Remark. Because $N_{\phi}$ is continuous off of a set of logarithmic capacity zero, we may rephrase Question 2 of the introduction as follows. Suppose that $N_{\phi}$ agrees with a continuous, radial, subharmonic function off of a set of logarithmic capacity zero; must $\phi$ be a constant multiple of an inner function?

\section{ACKNOWLEDGMENT}

The author would like to thank Joe Cima for helpful discussions and for sharing with him reference [2]. 


\section{ADDED IN PROOF}

Alec Matheson has pointed out to the author that a complete characterization of those subsets of $\left\{t \mapsto t^{p}: p>-1 / 2\right\}$ that span a dense subset of $L^{2}([0,1])$ may be found in Interpolation and Approximation by P. J. Davis (Dover, New York, 1975, p. 270). Davis attributes the characterization to Müntz.

\section{REFERENCES}

[1] Sheldon Axler, Paul Bourdon, and Wade Ramey, Harmonic function theory, Springer-Verlag (GTM 137), New York, 1992. MR 93f:31001

[2] Joseph A. Cima, Boris Korenblum, and Michael Stessin, "On Rudin's Orthogonality and Independence Problem", preprint.

[3] P. L. Duren, Theory of $H^{p}$ Spaces, Academic Press, New York, 1970. MR 42:3552

[4] M. Essén and D.F. Shea, "On some questions of uniqueness in the theory of symmetrization", Ann. Acad. Sci. Fennicae, Series A.I. Math, 4 (1978/79), 311-340. MR 81d:30002

[5] M. Essén, D.F. Shea, and C.S. Stanton, "A value-distribution criterion for the class $L \log L$, and some related questions", Ann. Inst. Fourier (Grenoble) 35 (1985), 127-150. MR 87e:30041

[6] J. E. Littlewood, "On inequalities in the theory of functions", Proc. London Math. Soc. (2) 23 (1925), 481-519.

[7] Walter Rudin, Real and Complex Analysis, 2nd edition, McGraw-Hill, New York, 1974. MR 49:8783

[8] J. H. Shapiro, "The essential norm of a composition operator", Annals of Math. 125 (1987), 375-404. MR 88c:47058

Department of Mathematics, Washington and Lee University, Lexington, Virginia 24450

E-mail address: pbourdon@wlu.edu 